\title{
Official Protocol Title
}

National Cancer Institute

\section{Source}

National Cancer Institute. Official Protocol Title. NCI Thesaurus. Code C132346.

The formal, descriptive name of the protocol. 